Urticarial manifestations associated with herpes simplex virus type 2

The association of certain dermatoses with herpes simplex virus (HSV) infection has long been appreciated. The best-documented cutaneous association of $\mathrm{HSV}$ is erythema multiforme because of the detection of HSV DNA in cutaneous lesions by using PCR. ${ }^{1}$ Urticaria may be precipitated by multiple factors including infectious agents such as hepatitis B and $C$ viruses. According to a series on additional diagnosis accompanying urticaria, ${ }^{2}$ the association with HSV has not been found in different studies.

A 58 year old woman presented with chronic urticaria for 6 weeks. At the beginning of the urticaria, treatment with oral prednisolone $(1 \mathrm{mg} / \mathrm{kg} /$ day $)$ and hydroxyzine (25 $\mathrm{mg} /$ day) completely cured the lesions in 24 hours. Four days after stopping her treatment, the urticaria recurred. Oral antihistamine and corticosteroids were ineffective. At the sixth week, the patient developed vesicular lesions localised on the low back, compatible with an herpes eruption. Nevertheless, she was on no regular medication, in good health and had a long history of genital herpes infection from the age of 23 years (one eruption per year). Local virological tests (ELISA and culture) performed on a vesicle showed HSV-2. Routine biological and biochemical screening tests, including haemoglobin, white cell count, erythrocyte sedimentation rate and liver function tests were normal. Syphilis and HIV serologies were negative. Histological examination of an urticarial lesion confirmed this diagnosis (oedema of the dermis, presence of eosinophils). Direct immunofluorescence examination was negative. The patient received treatment with acyclovir $250 \mathrm{mg}$ diluted in $50 \mathrm{ml}$ of isotonic $\mathrm{NaCl}$ by IV infusion spaced at 8 hours intervals. Six hours after the first infusion of acyclovir, she dramatically improved with a complete resolution of the urticaria. Acyclovir by IV infusion was given for 3 days and then orally for 10 days. The urticaria did not recur in the 8 months following the treatment.

Urticarial manifestations presented in this case appeared 6 weeks before the HSV lesions. The clinical appearance and histological features of the lesions were those of urticaria. This may be the predominant or sole feature of the prodromal serum-sicknesstype syndrome which occurs in 20 to $30 \%$ of patients with acute hepatitis $B$ virus (HBV) infection. ${ }^{3}$ The exact role which HSV may play in the pathogenesis of urticaria-HSV is unknown but it may be related to a hypersensitivity reaction. Deposition of immune complexes containing HBs antigen in involved cutaneous vessels as described in urticaria$\mathrm{HBV}^{4}$ have not been found in our case.

To our knowledge, no previous similar case of chronic urticaria preceding genital herpes has been described in the literature. M C MARGUERY

B PERIOLE P BAYLE J BAZEX
Département de Dermatologie Vénéréologie et Allergologie, Hôpital Purpan - place Baylac-31059, Toulouse Cédex, France

Address correspondence to Dr F El Sayed.

1 Brice SL, Krzemien D, Weston WL, et al. Detection of herpes simplex virus DNA in cutaneous lesions of erythema multiforme. F Invest Dermatol 1989;93:183-7.

2 Juhlin L. Recurrent urticaria: clinical investigation of 330 patients. Br $\mathcal{F}$ Dermatol 1981;104:369-81.

3 Sarkany I. Cutaneous manifestations of hepatobiliary disSarkany I. Cutaneous manifestations of hepatobiliary disDermatology in General Medicine. New York, McGrawDermatology in General Medicine.
Hill Inc. 3rd Ed 1987;2:1947-64.

4 Neuman HAM, Berretty PJM, Reinders Folmer SCC, et al. Hepatitis B surface antigen deposition in the blood vessel walls of urticarial lesions in acute hepatitis $\mathrm{B} . \mathrm{Br} \mathcal{F}$ Dermatol 1981;104:383-8.

\section{Azithromycin and syphilis}

Azithromycin, a new long-acting azalide antibiotic, became the standard treatment for uncomplicated genital chlamydial infection in the Northern Territory of Australia in July 1994. A trial of this agent in the treatment of genital donovanosis is also in progress with the approval of the responsible ethics committee. We have since recognised clinical or serological resolution of syphilis in two patients who happened to receive azithromycin for one of the above indications.

A 26 year old Aboriginal woman presented with vulval lesions consistent with donovanosis. She agreed to enrol in the trial. As stipulated in the study protocol, biopsy and other specimens were collected and azithromycin treatment $(500 \mathrm{mg}$ daily for seven days) commenced prior to confirmation of the diagnosis. Laboratory investigations subsequently revealed gonococcal cervicitis and a rapid plasma reagin (RPR) titre of $1: 32$. No Donovan bodies were seen in the biopsy and the histological appearance, particularly a dense perivascular plasmacytic infiltrate, was suggestive of a syphilitic lesion. Upon review one week later the vulval lesions had completely resolved. The patient was withdrawn from the trial because secondary syphilis was considered the likely diagnosis based on the rapid resolution of the lesions, the raised $R P R$ titre and the histological findings.

The second patient, a 32 year old Aboriginal man, took part in a screening programme for sexually transmitted diseases (STDs). He was asymptomatic and genital examination was normal. However, a leucocyte esterase dipstick test of his first void urine was positive suggesting urethritis. Urethral swab and venous blood specimens were collected and the patient given $1.0 \mathrm{~g}$ azithromycin orally. Laboratory investigations later confirmed gonococcal and chlamydial urethritis and found an RPR titre of 1:32. Eighteen months previously the patient's RPR titre had been $1: 1$. He was lost to follow-up for one month. When finally reviewed his RPR titre was 1:1 though he had received no other antibiotics since the single dose of azithromycin.

Patients presenting with STDs often have multiple infections. Hence some patients receiving the new regimen of a single $1.0 \mathrm{~g}$ dose of azithromycin for chlamydial or 
gonococcal genital infections will also have syphilis. ${ }^{12}$ Azithromycin has antitreponemal activity in vitro and in the rabbit model. ${ }^{34}$ Furthermore, Verdon et al $^{5}$ recently described the clinical efficacy of oral azithromycin (500 $\mathrm{mg}$ once daily for 10 days) for treating primary and secondary syphilis. Our experience with the first case supports their finding. More importantly, the serological resolution of early latent syphilis in the second patient suggests that the single $1.0 \mathrm{~g}$ dose of azithromycin used for urethritis and cervicitis is also effective.

This observation has important public health implications. While penicillin-based regimens must remain the treatment of choice until long-term studies demonstrate that azithromycin prevents the late sequelae of syphilis (both patients have since received $2 \cdot 4$ million units benzathine penicillin as definitive treatment), widespread use of single dose azithromycin for chlamydia and gonorrhoea may fortuitously reduce the transmission of syphilis by rendering patients with concurrent syphilis non-infectious.

$$
\begin{array}{r}
\text { F J BOWDEN } \\
\text { B FARMER } \\
\text { J BULLEN } \\
\text { V CHAMBERLAIN } \\
\text { AIDS/STD Unit, Block 4, } \\
\text { Royal Darwin Hospital, Rocklands Drive, } \\
\text { Casuarina, Northern Territory 0810, Australia } \\
\text { I BASTIAN } \\
\text { Menzies School of Health Research, } \\
\text { Casuarina, Darwin, Australia }
\end{array}
$$

Address correspondence to: Dr F J Bowden.

1 Martin DH, Mroczkowski TF, Dalu ZA, et al and the Azithromycin for Chlamydial Infections Study Group. A controlled trial of a single dose of azithromycin for the controlled trial of a single dose of azithromycin for the
treatment of chlamydial urethritis and cervicitis. $N$ Engl $f$ treatment of chlamydial

2 Waugh MA. Open study of the safety and efficacy of a single oral dose of azithromycin for the treatment of uncomplicated gonorrhoea in men and women. $f$ Antimicrob Chemother 1993;31 (suppl E): 193-8.

3 Stamm LV, Parrish EA. In-vitro activity of azithromycin and CP-63,956 against Treponema pallidum. $\mathcal{F}$ Antimicrob Chemother 1990;25 (suppl A):11-4.

4 Lukehart SA, Fohn MJ, Baker-Zander SA. Efficacy of azithromycin for therapy of active syphilis in the rabbit

model. F Antimicrob Chemother 1990;25 (suppl A):91-9. Verdon MS, Handsfield HH, Johnson RB. Pilot study of
azithromycin for treatment of primary and secondary azithromycin for treatment of primary
syphilis. Clin Infect Dis 1994;19:486-8.

\section{Syndromic management of genital ulcer disease-a critical appraisal}

Control of sexually transmitted diseases (STDs) is of paramount importance in the present era keeping in mind the risk of HIV transmission.

WHO recommends use of simple algorithms for syndromic management which are based on a constellation of signs and symptoms produced by different (or a majority) of the organisms causing each of these syndromes (WHO Technical Report Series, 810, Geneva, 1991). Need for syndromic management was felt because clinical diagnosis of STDs is not always correct and time taken by laboratory tests might delay the treatment, thus prolonging the period of infectivity.

We find one of the flow charts related to genital ulcer disease (GUD) quite impractical for the following reasons:

(1) Signs and symptoms may not be consistent or specific for diseases like transient chancroid, herpetic chancroid, chancroidal ulcers. Ulcers of lymphogranuloma venereum and herpes progenitalis might be impossible to differentiate even by the most experienced specialists. Presence of HIV could alter the typical morphology of all GUDs.

(2) Genital ulcers due to mixed infections might create a diagnostic and therapeutic dilemma.

(3) In developing countries GUD related lymphadenopathy is difficult to differentiate from lymphadenopathy following tuberculosis, leprosy and infected lesions over lower limbs in people who walk bare feet especially when the genital ulcer has healed.

(4) The flow chart does not address the problems of GUD in women, such as hidden GUD presenting as vaginal discharge, nor does it include treatment for sexual partners.

(5) Chemical ulcers following cleansing of the genital area with antiseptics like chlorhexidine $^{1}$ as a part of prophylactic behaviour may mimic GUD/balanoposthitis of bacterial or candidal aetiology. Treatment for presumed infective aetiologies (non-diseases) would be a huge waste of precious drugs in developing countries, morals and ethics apart. With even a faint possibility of drug resistance, the consequences could be serious. Similarly treatment for traumatic ulcers, fixed drug eruption, Behcet's disease and aphthosis etc, as for GUD such as syphilis or chancroid is uncalled for.

(6) The psychological trauma incurred by misdiagnosis of non-STDs as STDs might well cause serious disharmony in the conjugal life of patients.

(7) False labelling of disease would generate wrong epidemiological data. This would greatly hamper formulation of pragmatic STD control programmes for the future.

Hence we believe that the stress should be on: (1) Individual assessment of cases, (2) Proper clinical supervision and guidance of junior clinicians and paramedical staff, (3) Appropriate referral whenever required.

We would welcome views and suggestions of other physicians from developing countries.

BHUSHAN KUMAR
SANJEEV HANDA
GOUTAM DAWN
Department of Dermatology,
Venereology and Leprology,
PGIMER, Chandigarh-160 012, India

Address correspondence to: Dr Bhushan Kumar.

Accepted for publication 28 February 1995

1 Fisher AA. Allergic reactions to feminine hygiene sprays. Arch Dermatol 1973;108:801-2.

\section{An unusual cause of incontinence}

A 19 year old woman was referred by her general practitioner with a four week history of urinary incontinence. Leaking occurred only during coitus, and particularly on deep vaginal penetration. On a few occasions 